\title{
Study of Methods for Lowering the Lasing Frequency of a Terahertz Quantum-Cascade Laser Based on Two Quantum Wells
}

\author{
D. V. Ushakov ${ }^{a}$, Yu. G. Sadofyev ${ }^{b}$, and N. Samal ${ }^{c}$ \\ ${ }^{a}$ Belarussian State University, pr. Nezavisimosti 4, Minsk 220030 Belarus \\ e-mail: ushakovdv@bsu.by \\ ${ }^{b}$ Lebedev Physical Institute, Russian Academy of Sciences, Leninskii pr. 53, Moscow, 119991 Russia \\ ${ }^{c}$ Trion Technology, 85281 Tempe, AZ, USA \\ Submitted April 25, 2012; accepted for publication April 25, 2012
}

\begin{abstract}
Two mechanisms for achieving lower terahertz-range frequencies in quantum-cascade structures with two quantum wells based on GaAs/AlGaAs compounds are proposed. The first mechanism is based on the introduction of composite quantum wells consisting of a narrow $(\sim 2 \mathrm{~nm})$ quantum well with a low potential barrier, being within the main wide quantum well. The second mechanism is based on barriers with unequal heights, arranged in front of and behind the composite quantum well. Optimized quantum-cascade laser structures emitting in the regions of $\sim 2.15$ and $\sim 1.35 \mathrm{THz}$ are calculated.
\end{abstract}

DOI: $10.1134 / \mathrm{S} 106378261211022 \mathrm{X}$

\section{INTRODUCTION}

Recently, intersubband optical transitions in the terahertz range in semiconductor multi-period quantum-cascade lasers (QCLs) are actively studied in view of their great prospects for practical applications. All currently implemented THz-range QCLs are mainly developed on GaAs/AlGaAs structures operating on the principle of "resonant optical-phonon emission" [1-4], based on rapid depopulation of the lower operating level due to resonant optical-phonon emission. A single structure cascade can include four [1], three [2], or two [4] quantum wells ( $Q W)$ of different widths, separated by tunneling-transparent barriers. Accordingly, QCLs operate according to five- [1], four- [2], and three-level [4] schemes. The highest achieved operating temperatures for THz-QCLs are $117 \mathrm{~K}$ in the continuous mode [3] are $186 \mathrm{~K}$ for pulsed pumping [5] and $225 \mathrm{~K}$ in the case of additional use of a magnetic field [6]. The QCL radiation power in the frequency range of 3-4.5 THz reaches $100 \mathrm{~mW}$; however, when moving from 3 to $1 \mathrm{THz}$, the output powers decrease to $\sim 10 \mathrm{~mW}$.

In [4], $\mathrm{GaAs} / \mathrm{Al}_{0.15} \mathrm{Ga}_{0.85} \mathrm{As}$ quantum-cascade structures with two QWs, operating according to the three-level scheme, were proposed. As shown in Fig. 1, population inversion is reached between levels $3-2$, and 1'-2 during depopulation of level 2 due to resonant optical-phonon emission; in this case, level 3 is tunnelly related to level 1' of the previous cascade [4]. In such structures, lasing was achieved at a frequency of $4.6 \mathrm{THz}$, and the highest operating temperature of
$121 \mathrm{~K}$ was attained. It is complicated to extend the QCL with two QWs to lower frequencies, since it is not quite possible to vary the thicknesses of the quantum wells and barrier layers (there are only four of them) due to the necessity to simultaneously satisfy the con-

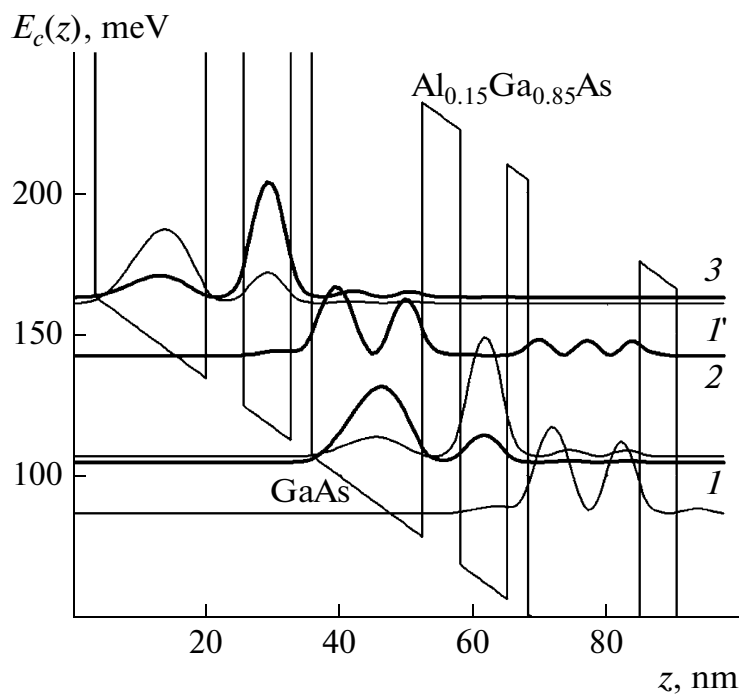

Fig. 1. Conduction band diagram $E_{c}(z)$ and squared magnitudes of the electron wave functions in the electric field $E=17.3 \mathrm{kV} / \mathrm{cm}$. The structure layer thicknesses are (from left to right) $3.1 / 16.7 / 5.6 / 7.1 \mathrm{~nm}$ (in the layer sequence, the GaAs quantum well is shown in bold, the underlined layer is doped with a $\mathrm{Si}$ impurity with a layer concentration of $2.17 \times 10^{10} \mathrm{~cm}^{-2}$ ). 
Parameters of the band structure of the semiconductors

\begin{tabular}{l|l|l|l|l|l|c|c|c|c|c}
\hline \multicolumn{1}{c|}{ Compound } & $m_{c} / m_{e}$ & $\gamma_{1}$ & $\gamma_{2}$ & $\gamma_{3}$ & $E_{p}, \mathrm{eV}$ & $\Delta S_{0}, \mathrm{meV}$ & $E_{g 0}, \mathrm{eV}$ & $\alpha, \mathrm{meV} / \mathrm{K}$ & $\beta, \mathrm{K}$ & $-F$ \\
\hline $\mathrm{GaAs}$ & 0.067 & 6.98 & 2.06 & 2.93 & 28.8 & 341 & 1.519 & 0.5405 & 204 & 1.938 \\
$\mathrm{AlAs}$ & 0.15 & 3.76 & 0.82 & 1.42 & 21.1 & 280 & 3.099 & 0.885 & 530 & 0.477 \\
$\mathrm{Al}_{0.02} \mathrm{Ga}_{0.98} \mathrm{As}$ & 0.069 & 6.96 & 2.04 & 2.90 & 28.6 & 340 & 1.553 & 0.547 & 211 & 1.909 \\
$\mathrm{Al}_{0.15} \mathrm{Ga}_{0.85} \mathrm{As}$ & 0.079 & 6.50 & 1.87 & 2.70 & 27.6 & 332 & 1.747 & 0.592 & 253 & 1.719 \\
$\mathrm{Al}_{0.19} \mathrm{Ga}_{0.81} \mathrm{As}$ & 0.083 & 6.37 & 1.82 & 2.64 & 27.3 & 329 & 1.800 & 0.606 & 265.9 & 1.660 \\
\hline
\end{tabular}

Note: $m_{c}$ is the electron effective mass and $m_{e}$ is the free electron mass.

ditions of resonant optical-phonon emission and resonant tunneling.

This paper is devoted to numerical calculations of the energy and spectral characteristics of QCLs with two QWs and to the search for ways to extend the QCL to lower frequencies of the $\mathrm{THz}$ range by varying the component composition of the quantum wells and barrier layers.

\section{THEORETICAL MODEL FOR CALCULATING ENERGY LEVELS AND AMPLIFICATION SPECTRA}

The energies and wave functions were determined using the extended Bastard model by solving the Schrödinger equations $[7,8]$

$$
\begin{aligned}
& -\frac{h^{2}}{2} \frac{d}{d z}\left[\frac{1}{m_{c}\left(z, E_{f}\right)} \frac{d \psi_{f}(z)}{d z}\right] \\
& -\left[E_{f}-E_{c}(z)\right] \psi_{f}(z)=0,
\end{aligned}
$$

where $E_{f}$ are the size-quantization levels, $\psi_{f}(z)$ are the wave-function envelopes, and $E_{c}(z)$ is the profile of the potential energy of the conduction band in the electric field. The energy and coordinate dependence of the effective mass $m_{c}(E, z)$ was determined according to [7] as

$$
\begin{gathered}
\frac{m_{e}}{m_{c}(E, z)}=1+2 F(z) \\
+\frac{E_{p}}{3}\left[\frac{2}{E+E_{c}(z)}+\frac{1}{E+E_{c}(z)+\Delta S_{0}(z)}\right] .
\end{gathered}
$$

The band-structure parameters for ternary compounds $\mathrm{A}_{x} \mathrm{~B}_{1-x} \mathrm{C}$ were approximated by experimental and theoretical data for binary $\mathrm{AB}, \mathrm{AC}$, and $\mathrm{BC}$ compounds [9] using the quadratic approximation parameter $b_{\mathrm{ABC}}$

$$
G_{\mathrm{ABC}}(x)=x G_{\mathrm{AC}}+(1-x) G_{\mathrm{BC}}-x(1-x) b_{\mathrm{ABC}} .
$$

Here, instead of $G$, the Luttinger parameters $\left(\gamma_{1}, \gamma_{2}, \gamma_{3}\right)$, the energy related to the Kane matrix element of interband transitions $E_{p}$, the spin-orbit splitting energy $\Delta S_{0}$, the band gap $E_{g 0}$, Varshni parameters $\alpha$ and $\beta$, and the parameter $F$ characterizing the interaction of the conduction band with the overlying energy bands should be substituted. The interpolated characteristics necessary to calculate the band structure of $\mathrm{Al}_{1-x} \mathrm{Ga}_{x}$ As compounds are listed in the table. The quadratic approximation parameter $b_{\mathrm{ABC}}$ was chosen as 0.7165 in calculating $E_{g 0}$ and 0 in calculating other parameters.

The filling factor of the energy levels and the corresponding quasi-Fermi levels for the steady-state case were determined by numerically solving the set of balance equations [10]

$$
\begin{aligned}
& \frac{d n_{i_{N}}}{d t}=\sum_{j_{N} \neq i_{N}}\left(R_{j_{N} i_{N}}-R_{i_{N} j_{N}}\right)+\sum_{j_{N-1}}\left(R_{j_{N-1} i_{N}}-R_{i_{N} j_{N-1}}\right) \\
& +\sum_{j_{N+1}}\left(R_{j_{N+1} i_{N}}-R_{i_{N} j_{N+1}}\right), \\
& \sum_{i_{N}=1}^{n_{\max }} n_{i_{N}}=n_{\text {stot }} .
\end{aligned}
$$

The set of balance equations (4) includes terms corresponding to transition rates within the $N$-th cascade and between $(N-1)-N$ and $N-(N+1)$ cascades. The latter equation includes the sum of electron densities $n_{i_{N}}$ at all levels of the $N$-th cascade, equal to $n_{\text {stot }}$ and defined by layer doping levels. In calculating the resulting transition rates, scattering at optical phonons and electron-electron scattering [10] were taken into account.

The gain $g$ for intrasubband transitions, depending on the light frequency $v$, was determined in the multilevel approximation [11] 

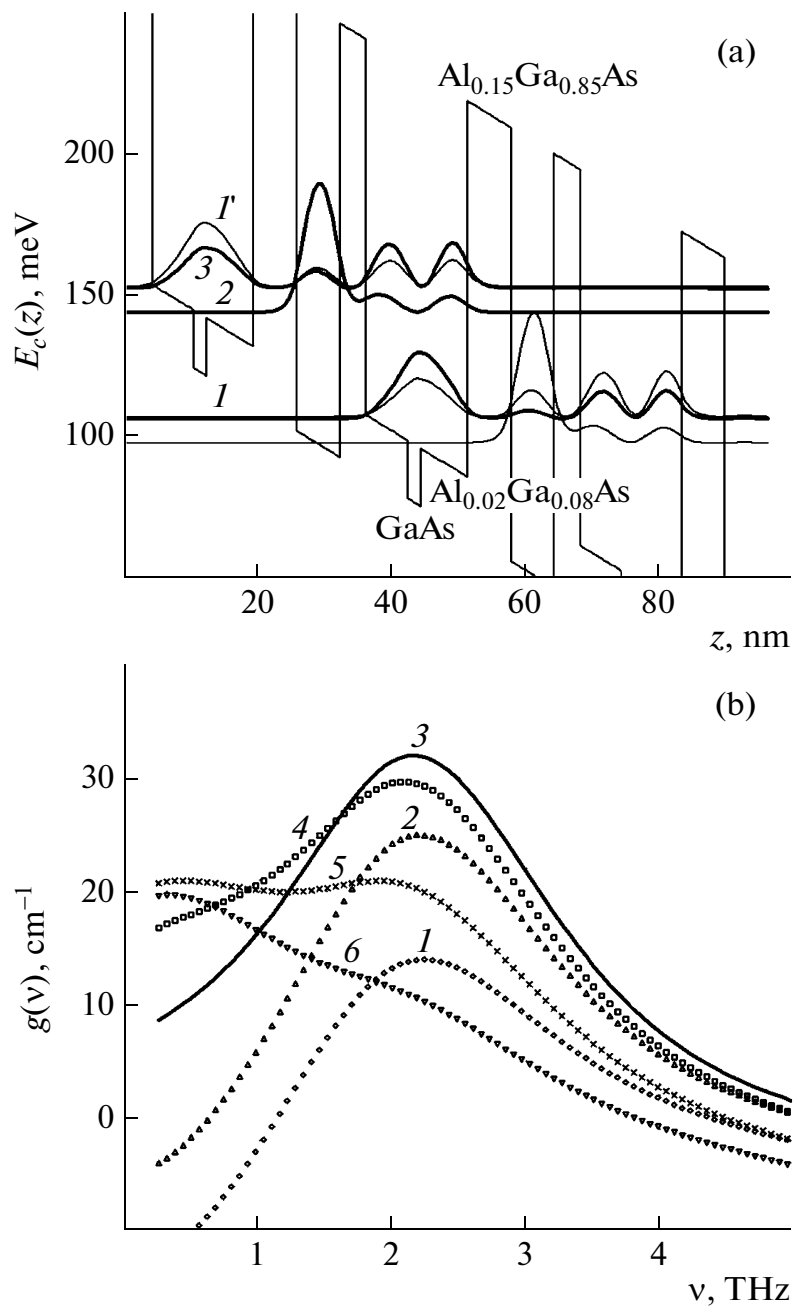

Fig. 2. (a) Conduction band diagram $E_{c}(z)$ and squared magnitudes of the electron wave functions for a structure with identical barrier layers $\mathrm{Al}_{0.15} \mathrm{Ga}_{0.85} \mathrm{As}$ in the electric field $E=14.35 \mathrm{kV} / \mathrm{cm}$ and (b) amplification spectra at $E=$ (1) 14.15 , (2) 14.25 , (3) 14.35 , (4) 14.45 , (5) 14.55 , and (6) $14.65 \mathrm{kV} / \mathrm{cm}$.

$$
\begin{aligned}
g(v)= & \sum_{n} \sum_{m} g_{n m}(v)=\frac{e^{2}}{h^{3} \varepsilon_{0} c n_{r} d} \sum_{n} \sum_{m} m_{f m}\left|z_{n m}\right|^{2} \\
& \times \int_{E_{f m}}^{\infty} d E_{f}\left(E_{i}-E_{j}\right) F\left(h v-\left(E_{i}-E_{f}\right), \gamma\right) \\
& \times\left[f\left(E_{i}-F_{n}\right)-f\left(E_{f}-F_{m}\right)\right],
\end{aligned}
$$

where $E_{i}=\left(E_{f}-E_{f m}\right)\left(m_{f m} / m_{i n}\right)+E_{i n} ; z_{n m}=\int \psi_{n}^{*} z \psi_{m} d z$ is the matrix element of the dipole transitions; $F_{n}$ and $F_{m}$ are the quasi-Fermi levels in the minibands $n$ and $m$ with the energies $E_{i n}, E_{f m}$ and the effective masses $m_{i n}$, $n_{f m} ; d$ is the quantum-cascade structure thickness; $n_{r}$ is the refractive index; and $F(\Delta E)$ is the function describing the profile of emission-line broadening [11]. Summation in (5) is performed over all quantum numbers of the initial $(n)$ and final $(m)$ states for which $E_{i n}-E_{f m}>0$.

Previously, this technique was successfully tested at numerical calculations of QCLs with four and three QWs, grown by molecular-beam epitaxy by Trion Technology (Tempe, Arizona, USA) [12]. Figure 1 also shows the test calculations of the band structure, energy levels, and wave functions of QCLs based on GaAs- $\mathrm{Al}_{0.15} \mathrm{Ga}_{0.85} \mathrm{As}$ with two QWs, proposed in [4]. In the calculations, the potential barrier height in the conduction band was set to $\Delta E_{c}=153.7 \mathrm{meV}$. As numerical calculations show, the main transitions occur between the 3-2 and 1'-2 levels in the region of $\sim 4.6 \mathrm{THz}$, which is in good agreement with the experimental data of [4].

\section{DISCUSSION OF WAYS OF EXTENSION TO LOWER FREQUENCIES OF THE TERAHERTZ RANGE}

One of the ways to obtain additional degrees of freedom to extend to lower frequencies of the $\mathrm{THz}$ range can be minor changes in the molar composition of the QWs and the introduction of composite QWs with narrow QWs $(\sim 2 \mathrm{~nm})$ bounded by a low potential barrier located within the wide QW. The latter allows efficient control of the energy position of the ground state of size quantization of the composite QW.

Figure 2a shows the optimized design of such a QCL type. A narrow $\mathrm{GaAs} / \mathrm{Al}_{0.02} \mathrm{Ga}_{0.98} \mathrm{As} \mathrm{QW}$ of the depth $\Delta E_{c}=20.5 \mathrm{meV}$ is embedded into the structure of a wide $\mathrm{Al}_{0.02} \mathrm{Ga}_{0.98} \mathrm{As} / \mathrm{Al}_{0.15} \mathrm{Ga}_{0.85} \mathrm{As} \mathrm{QW}$ of the depth $\Delta E_{c}=133.7 \mathrm{meV}$. The layer thicknesses of the structure from left to right are $/ 14 / 22 / 7 / 25 / 23 / \underline{\mathbf{2 3}}$ monolayers. In the layer sequence, the GaAs quantum wells are shown in bold; $\mathrm{Al}_{0.02} \mathrm{Ga}_{0.98} \mathrm{As}$ are in bold italic. The underlined layers are doped with $\mathrm{Si}$ impurities with a layer concentration of $2.17 \times 10^{10} \mathrm{~cm}^{-2}$. We note that all QCL layer thicknesses are multiples of the GaAs and $\mathrm{AlGaAs}$ monolayer thickness $(0.2825 \mathrm{~nm})$, which provides better quality heterojunctions, high growth rates, and higher expected operating temperatures [13].

The introduction of a narrow QW into the wide well makes it possible to lower the radiation energy by changing the position of the size-quantization states of the composite QW with respect to the ground state of the narrower (23 monolayers) $\mathrm{GaAs} / \mathrm{Al}_{0.15} \mathrm{Ga}_{0.85} \mathrm{As}$ QW. We note that in the structure version of [4] (Fig. 1), the 3-2 and 1'-2 transitions occur between the energy level 3 (ground level) of the narrow QW and the energy level 2 (excited level) of the wide QW and between the first energy level 1' (ground level) of the wide QW of the previous cascade and the energy level 2 
of the wide QW of the next cascade. In the design we proposed (Fig. 2a), the 3-2 and 1'-2 transitions occur between the energy level 3 (excited level) of the composite QW and the energy level 2 (ground level) of the narrow QW and between the energy level 1' (ground level) of the composite QW of the previous cascade and the energy level 2 of the narrow QW of the next cascade.

The introduction of a narrow QW into the wide well centers the wave function and makes it possible to control the matrix element of the dipole transitions between the operating levels 3-2 at a fixed electric field strength $E=14.5 \mathrm{kV} / \mathrm{cm}$ and constant thickness of the composite wide QW. If the narrow QW seven monolayers thick is displaced to the left edge of the composite QW, the transitions between the operating levels become more straight, and the matrix elements of the dipole transitions increase from $z_{32}=4.82 \mathrm{~nm}$ for the composite QW $(22 / 7 / 25)$ to $z_{32}=5.54 \mathrm{~nm}$ for the displacement of five monolayers to the left $(17 / 7 / 30)$. In the case of displacements to the right edge of the composite QW, the transitions become more diagonal and the matrix elements of the dipole transitions decrease, $z_{32}=2.94 \mathrm{~nm}$ for the composite QW (30/7/17).

Figure $2 \mathrm{~b}$ shows the calculated amplification spectra of such structures. The gain maximum increases and shifts to lower frequencies with the electric field strength. At $E=14.35 \mathrm{kV} / \mathrm{cm}$, the best conditions for resonant tunneling are achieved (levels 3 and 1' coincide). In this case, the gain is $32 \mathrm{~cm}^{-1}$ at a frequency of $\sim 2.15 \mathrm{THz}$, which is significantly smaller than in [4].

It is interesting to study the effect of the height of the narrow barrier layer in front of the wide composite QW on the amplification spectra at fixed layer thicknesses, which is possible by varying the molar composition of the barrier layer. The QCL characteristics were calculated for the $\mathrm{Al}_{x} \mathrm{Ga}_{1-x} \mathrm{As}$ barrier layer $3.955 \mathrm{~nm}$ (14 monolayers) thick at $x$ from 0.09 to 0.19 , which corresponds to potential barrier heights from 92.2 to $194.7 \mathrm{eV}$. The calculations showed that the distance between the levels $2-1$ decreases at fixed structure layer thicknesses and $x<0.15$, and becomes smaller than the optical-phonon frequency, which results in lasing failure. At $x>0.15$, the distance between the levels $2-1$ increases, but is controlled by the electric field and insignificantly differs from the optical-phonon frequency. Figure 3 shows the results of calculations for the $\mathrm{Al}_{0.19} \mathrm{Ga}_{0.81}$ As barrier layer. As seen in Fig. 3b, the behavior of the gain maximum with increasing electric field strength is similar to that shown in Fig. 2b, but already at higher $E$. The best conditions for resonant tunneling are achieved at the electric field strength $E=14.55 \mathrm{kV} / \mathrm{cm}$. In this case, the gain is $23 \mathrm{~cm}^{-1}$ at a frequency of $\sim 1.35 \mathrm{THz}$. The
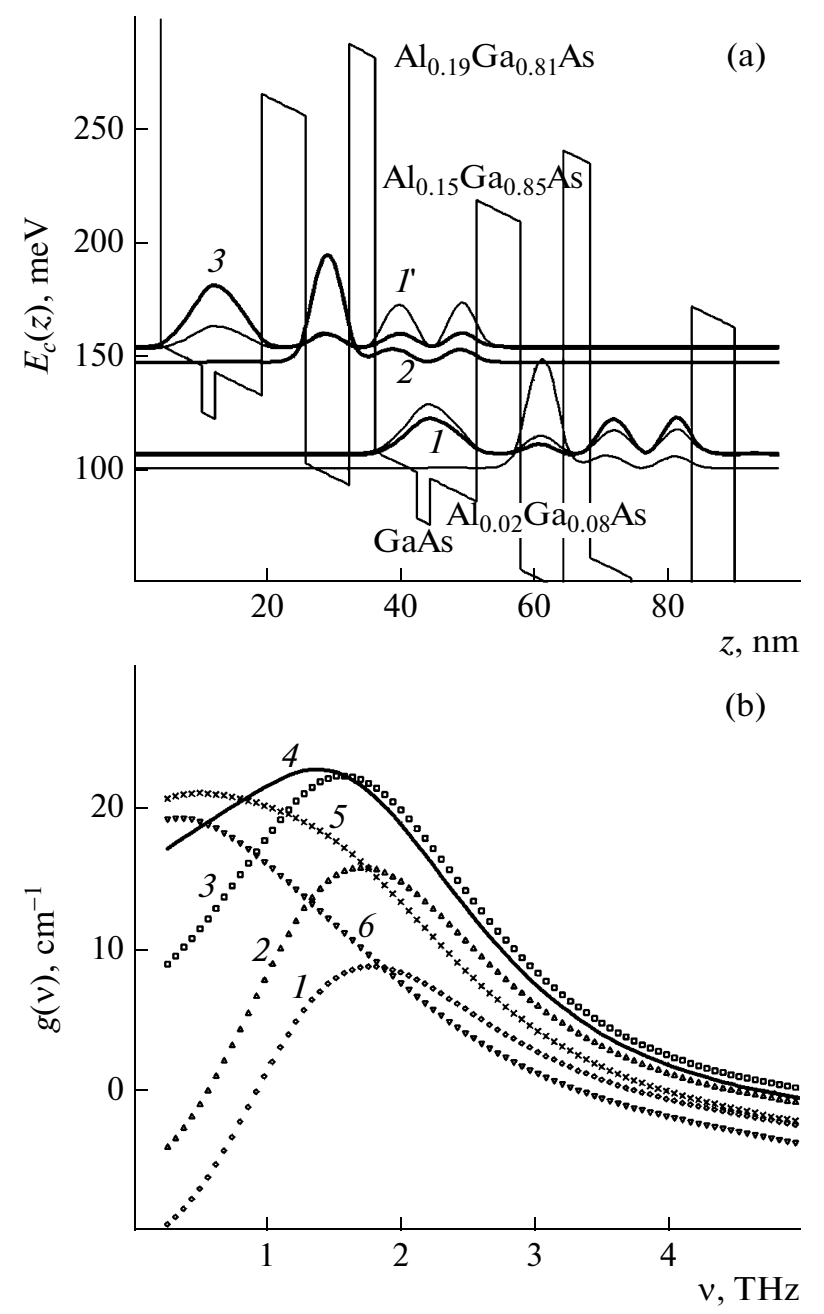

Fig. 3. (a) Conduction band diagram $E_{c}(z)$ and squared magnitudes of the electron wave functions for a structure with different barrier layers, $\mathrm{Al}_{0.19} \mathrm{Ga}_{0.81} \mathrm{As}$ in front of the wide composite quantum well and $\mathrm{Al}_{0.15} \mathrm{Ga}_{0.85}$ As behind it, in the electric field $E=14.55 \mathrm{kV} / \mathrm{cm}$ and (b) amplification spectra at $E=$ (1) 14.25 , (2) 14.35 , (3) 14.45 , (4) $14.55,(5) 14.65$, and (6) $14.75 \mathrm{kV} / \mathrm{cm}$.

lower gains are caused by a small deviation from the condition of resonant optical-phonon emission.

\section{CONCLUSIONS}

A QCL design with two QWs was proposed, in which a narrow GaAs QW is embedded into a wide $\mathrm{Al}_{0.02} \mathrm{Ga}_{0.98} \mathrm{As} / \mathrm{Al}_{0.15} \mathrm{Ga}_{0.85} \mathrm{As} \mathrm{QW}$ structure. The maximum gain was achieved at a frequency of $\sim 2.15 \mathrm{THz}$. The matrix element of the dipole transitions for the operating lasing levels 3-2 can be controlled by varying the narrow QW position in the wide QW. An increase in the barrier-layer height in front of the wide composite QW results in a decrease in the emission frequency to $\sim 1.35 \mathrm{THz}$. The proposed ways, both 
together and individually, make it possible to introduce additional degrees of freedom to extend to the region of lower THz-range frequencies.

\section{ACKNOWLEDGMENTS}

D.V. Ushakov acknowledges the support of the State program of Scientific Research "Electronics and Photonics" (program no. 1.2.04) and the Belarussian Republican Foundation for Basic Research under the Belarussian-Russian Foundation for Basic Research (project no. F10R-091).

\section{REFERENCES}

1. B. S. Williams, S. Kumar, Q. Hu, and J. L. Reno, Electron. Lett. 42, 89 (2006).

2. H. Luo, S. R. Laframboise, Z. R. Wasilewski, G. C. Aers, and H. C. Liu, Appl. Phys. Lett. 90, 041112 (2007).

3. B. S. Williams, Nature Photon. 1, 517 (2007).

4. S. Kumar, C. W. I. Chan, Q. Hu, and J. Reno, Appl. Phys. Lett. 95, 141110 (2009).

5. S. Kumar, Q. Hu, and J. Reno, Appl. Phys. Lett. 94, 131105 (2009).
6. A. Wade, G. Fedorov, D. Smirnov, S. Kumar, B. S. Williams, Q. Hu, and J. L. Reno, Nature Photon. 3, 41 (2009).

7. K. H. Yoo, L. R. Ram-Mohan, and D. F. Nelson, Phys. Rev. B 39, 12808 (1989).

8. D. V. Ushakov and I. S. Manak, Opt. Spectrosc. 104, 767 (2008).

9. I. Vurgaftman, J. R. Meyer, and L. R. Ram-Mohan, J. Appl. Phys. 89, 5815 (2001).

10. D. V. Ushakov and I. S. Manak, J. Appl. Spectrosc. 74, 892 (2007).

11. D. V. Ushakov, V. K. Kononenko, and I. S. Manak, Quantum Electron. 40, 195 (2010).

12. A. V. Antonov, V. I. Gavrilenko, A. V. Ikonnikov, K. V. Maremyanin, A. A. Lastovkin, S. V. Morozov, D. V. Ushakov, Yu. G. Sadofyev, and N. Samal, Radiophys. and Quantum Electron. 52, 494 (2009).

13. Yu. G. Sadofyev, in Proceedings of the All-Russia Symposium on Nanophysics and Nanoelectronics (Nizh. Novgorod, 2011), Vol. 1, p. 244.

Translated by A. Kazantsev 